\title{
Resilience at Information Processing Level in Older Adults: Maintained Attention for Happy Faces when Positive Mood is Low
}

\author{
Ineke Demeyer ${ }^{1}$, Inke Urbain ${ }^{2}$, and Rudi De Raedt ${ }^{1}$ \\ Ghent University (Belgium) \\ WZC OLV van Lourdes Wakken VZW (Belgium)
}

We would like to thank the WZC O.L.V van Lourdes in Wakken for their collaboration with the recruitment and testing of their residents. This work was supported by a Grant BOF10/GOA/014 for a Concerted Research Action of Ghent University awarded to Rudi De Raedt and by a grant for an interuniversity research group PIPO [I/00125/01].

Correspondence concerning this article should be addressed to Ineke Demeyer. Ghent University. Department of Experimental Clinical and Health Psychology. Henri Dunantlaan 2, B-9000. Ghent (Belgium). Phone: +329-2649411. Fax: +329-2646489. E-mail: Ineke.Demeyer@UGent.be 


\begin{abstract}
Even though ageing is associated with declining cognitive capabilities, research has demonstrated an age-related improvement in affective well-being. This improvement can be related to increased resilience, developing as changes in emotion regulation at informationprocessing level. During negative mood, emotion regulation becomes a priority as demonstrated by an increased preference for positive over negative information in older adults. However, the effect of a positive mood on older adult's attentional preferences has not been established yet. To investigate this, 37 older adults were randomly assigned to a relaxation or a control condition (music). Mood state was assessed before and after the manipulation. Attentional bias was measured by an exogenous cueing task, in which the location of the target was correctly or incorrectly cued by happy, sad or neutral facial pictures. Both groups showed a decrease in negative $\operatorname{mood}(p<.001,95 \%$ CI $[2.73,5.97], d=.82)$ without changes in positive mood. The relaxation group showed a significantly bigger increase in feeling relaxed $\left(p=.017, \eta^{2} p=.15\right)$. No significant group differences were found for attentional bias. However, over the whole group, less positive mood after the manipulation was associated with more maintained attention for positive information $(r=-.49, p<.01)$. These results indicate that older adults deploy emotion regulation strategies in attention during low positive mood. Flexible attentional processing of emotional information might serve as a resilience factor to maintain well-being during later stages of life.
\end{abstract}

Keywords: attentional bias, older adults, positive mood, relaxation, resilience. 
Ageing is associated with a decline in cognitive capabilities, such as processing speed, problem solving and memory (Deary et al., 2009). Moreover, older adults are increasingly confronted with loss experiences, such as the loss in physical abilities and the deaths of loved ones. Unexpectedly, research has demonstrated an age-related improvement in affective wellbeing (e.g., Carstensen et al., 2011) indicating that older adults show resilience towards these negative circumstances. According to the socio-emotional selectivity theory (Carstensen, Isaacowitz, \& Charles, 1999), older adults allocate more resources towards emotion regulation because they prioritize current emotional wellbeing due to a more limited future time perspective. Thus, older adults' resilience may result from changes in emotion regulation.

One possible way for older adults to achieve current wellbeing is through emotion regulation at information-processing level (Mather \& Carstensen, 2005). In contrast to younger adults, older adults show an increased preference for positive information over negative information. This bias has been called 'the positivity effect' (Carstensen \& Mikels, 2005). Several studies have investigated differences in attentional processing of emotional information between younger and older adults. Even though there are many reports confirming a positivity effect in older adults' attentional processing compared to younger adults (e.g., Isaacowitz, Wadlinger, Goren, \& Wilson, 2006; Mather \& Carstensen, 2003), there are also several studies that could not find any difference in attentional processing of emotional information between younger and older adults (e.g., Steinmetz, Muscatell, \& Kensinger, 2010). A large metaanalysis by Murphy and Isaacowitz (2008) with 1085 older adults and 3150 younger adults led to the conclusion that emotional information processing remains stable over the course of life. In light of these inconsistent results, it has been highlighted that the positivity effect in emotional attention would be more pronounced under certain conditions (e.g., Urry \& Gross, 2010), which was confirmed by a more recent meta-analysis (Reed, Chan, \& Mikels, 2014).

Given that the positivity effect in attention is thought to reflect an emotion regulation strategy, it has been suggested that it might be influenced by the emotional state of the older adults (Isaacowitz \& Blanchard-Fields, 2012). More specifically, the positivity effect should become more pronounced when emotional well-being is threatened and emotion regulation is needed, such as during elevated negative mood. Even though there is an abundant literature regarding the impact of emotion on attentional processing in younger adults (for reviews, see Cisler \& Koster, 2010; De Raedt \& Koster, 2010), only a few studies have investigated the effects of emotional state in the field of age-related attentional biases. Demeyer and De Raedt (2013) showed that older adults who experienced more anxiety demonstrated more attentional avoidance of negative stimuli. Moreover, in a study investigating attention through eye tracking 
after a mood manipulation in both young and older adults (Isaacowitz, Toner, Goren, \& Wilson, 2008), it was also found that older adults show mood-incongruent gaze patterns. In contrast to younger adults who demonstrated mood-congruent gaze patterns (i.e., more time spent looking at negative stimuli during negative mood), older adults spent more time looking to positive stimuli and less time looking to negative stimuli during negative mood. These studies emphasize the importance of emotional state (i.e., negative mood, anxiety and stress) when investigating information processing in older adults. Moreover, they indicate that the positivity effect in attentional processing might serve emotion regulation during negative mood.

Importantly, the socioemotional selectivity theory (Carstensen et al., 1999) states that the goal of emotional wellbeing through emotion regulation becomes a priority during late life, but it also specifies that other goals, such as knowledge-related goals in which attention needs to be given to negative information, remain present in this age group (Carstensen, Fung, \& Charles, 2003). It is only when these goals conflict with current wellbeing that emotion-related goals will receive priority. In light of this reasoning, other goals may drive information processing of emotional information in older adults when emotion regulation is not needed, such as during a calm or positive mood. Although studies have provided evidence showing that negative mood leads to emotion regulation through mood-incongruent attentional processing (e.g., Demeyer \& de Raedt, 2013; Isaacowitz et al., 2008), little is known about older adults' information processing when emotional well-being is not threatened and emotion regulation is not needed, such as during a calm relaxed state. Given that the cognitive nature of experimental paradigms may activate negative mood in older adults through negative age-related stereotypes about their abilities to succeed in these tasks (Isaacowitz et al., 2008), we decided to use a relaxation procedure to induce such a calm state.

We aimed to reduce negative mood and possible stress caused by the test situation. In this way, we could assess the difference between a natural occurring mood context versus a calm and relaxed emotional state. In a between subjects design, older adults receiving a relaxation session were compared to a control group listening to music. Relaxation is a technique which is known to reduce negative mood states such as stress and anxiety, and to increase feelings of calmness, also in older adults (Sharpe, Williams, Granner, \& Hussey, 2007). This manipulation aimed at reducing the naturally occurring inter-individual variability in emotional state in order to test all older adults within the experimental group in a similar nonanxious or non-stressful mood context. The effectiveness of this manipulation was measured based on self-reported mood. 
To investigate the influence of the relaxation manipulation on attentional bias, we used an emotional variant of the exogenous cueing task (Koster, De Raedt, Goeleven, Franck, \& Crombez, 2005; Posner, 1980). In contrast to most frequently used paradigms in older adult research (i.e., the dot probe), this task allows distinction between maintained attention and attentional avoidance of emotional information. Even though attention research stresses the importance of differentiation between interrelated components of attention (Posner, 1980), up to date it remains unclear which components drive attentional processing of emotional information in older adults. The task was adjusted for older adults in Demeyer and De Raedt (2013) and uses long cue presentations because attentional biases in older adults have mainly been found in later stages of information processing (Isaacowitz, Allard, Murphy, \& Schlangel, 2009). As cues, this task uses both positive and negative information, which are represented by happy and sad faces.

The general aim of the present study was to investigate the influence of a calm, positive mood on attentional processing as measured by the exogenous cueing task. First, we explored the influence of the relaxation manipulation on attentional bias. If the positivity effect is most pronounced during a negative mood context (Demeyer \& De Raedt, 2013; Isaacowitz et al., 2008), we might anticipate to find group differences in attentional bias, showing less maintained attention to positive information or less attentional avoidance of negative information in the relaxation group compared to the control group. Secondly, we also examined the direct relationship between inter-individual differences in mood and attentional bias over the whole group to verify whether participants scoring lower on positive mood and/or higher on negative mood would show more attentional bias towards positive information and less attention towards negative information.

\section{Method}

\section{Participants}

This study was approved by the University's ethics committee. 37 older adults of Western European descent participated in this study. They were recruited at the rest-home where they resided. Participants were randomly divided in two groups. One group received relaxation and consisted of 19 participants (15 female, 4 male) with a mean age of 80.53 ( $S D=$ 8.70, range 60-93). The control group was comprised of 18 participants (13 female, 5 male) with a mean age of $80.50(S D=6.56$, range 66-90).

\section{Materials}

Mini Mental State Examination (MMSE). 
To screen for cognitive impairment, the MMSE was used (Folstein, Folstein, \& McHugh, 1975). The total MMSE scores range from 0 to 30, with higher scores indicating better cognitive functioning. Only participants scoring above the cut-off of 24 (indicating that there were no signs of cognitive decline, see Tombaugh \& McIntyre, 1992) were included in the study.

\section{Positive and negative affect schedule (PANAS).}

The PANAS (Watson, Clark, \& Tellegen, 1988) measures positive (PA) and negative affect (NA). In the state version, participants are asked to rate the extent to which they experience a mood state at the current moment on a scale ranging from 1 to 5 . The Dutch translation of the PANAS has shown good psychometric properties (Peeters, Ponds, \& Vermeeren, 1996).

\section{Visual Analogue Scale (VAS).}

We used VAS to assess whether the mood induction was successful. Using these scales, we assessed 6 statements: 'I feel relaxed/ calm/ pleasant/ tense/ nervous/ uncomfortable'. Participants indicate their level of agreement with each statement by placing a mark on a $100 \mathrm{~mm}$ line. This line is labelled on opposite ends: 'not at all' at the left and 'completely true' at the right. Responses are scored by measuring the distance from the left of the line to the marked point with ' 0 ' being the lowest score and ' 100 ' being the highest score. The 3 scores on the negative scales were reversed and a mean VAS score for relaxation was created by averaging the 6 scales. A higher mean VAS score reflects more relaxation. In this sample, the scale demonstrated good internal consistency with a Cronbach's Alpha of .85.

Exogenous Cueing Task (ECT).

The ECT examines attentional bias towards negative and positive information. Sixty coloured pictures of emotional faces (20 happy, 20 neutral and $20 \mathrm{sad}$ ) were selected from the Karolinska Directed Emotional Faces database (KDEF) based on a prior validation (Goeleven, De Raedt, Leyman, \& Verschuere, 2008). Inclusion criteria for the pictures were correct categorisation (> 90\% for happy, $>85 \%$ for sad, $>80 \%$ for neutral) and average rating of how well a picture displayed the emotion on a 9 point scale $(M=5.28$ for neutral, $M=6.78$ for happy, $M=6.02$ for sad). Moreover, a previous study showed that the pictures had similar ratings on both criteria in older adults (Demeyer \& De Raedt, 2013). All pictures were adjusted to the same size ( $326 \times 326$ pixels) and cut to exclude interference of background stimuli (hair, clothing).

The ECT was programmed using INQUISIT Millisecond software (De Clercq, Crombez, Buysse, \& Roeyers, 2003). All stimuli were presented against a black background. 
At the beginning of each trial, two white placeholders $\left(75 \mathrm{~mm}\right.$ by $75 \mathrm{~mm}$, visual angle: $\left.7.15^{\circ}\right)$ appeared on both sides of a fixation cross. These remained on screen during the entire trial. The middle of each of these frames was at $40 \mathrm{~mm}$ distance $\left(3.81^{\circ}\right.$ visual angle) from the fixation cross. 500ms later, one of the frames was replaced by a pictorial cue for $1000 \mathrm{~ms}$. Immediately after this cue disappeared, a target (a black square, $10 \mathrm{~mm}$ by $10 \mathrm{~mm}$, visual angle $1^{\circ}$ ) was presented in the middle of one of the two placeholders. This target remained on screen until response. Participants were asked to indicate as quickly and accurately as possible the location of this target by pressing the left or right button of a response box. It was emphasized that attention should be directed towards the fixation cross during the entire experiment. Participants got acquainted with the ECT during 16 practice trials. Then they performed the test block of 240 trials. All participants were seated at $60 \mathrm{~cm}$ viewing distance of the computer screen (a 19inch colour monitor).

In half of the trials, the location of the picture cued the location of the target correctly (valid trials). In the other $50 \%$ of the trials, the target was incorrectly cued (invalid trials). Participants were informed that the target location could not be predicted from the cue location. Pictures were presented randomly with an equal number of presentations for happy, sad and neutral faces and trial type (valid versus invalid). Additionally, 24 trials in which the fixation cross was briefly $(150 \mathrm{~ms})$ replaced by an arrow were inserted to control for central fixation and avoid that participants adopt a strategy of only fixating at one side of the screen. Participants were asked to indicate whether the arrow pointed left or right by pressing the left or right button of the response box.

\section{Procedure}

The residents of the rest-home were informed about the experiment and all volunteers were tested on their cognitive abilities with the MMSE. All volunteers were invited to participate, but those who scored below cut-off for the MMSE only received a relaxation session and were not included in the experiment. The other participants were randomly assigned to the relaxation group or the control group. All participants were tested individually in the relaxation room of the rest-home. At the beginning, written informed consent was obtained and participants filled in the questionnaires. In the relaxation group, each participant received a relaxation session that was guided by a licensed relaxation therapist. This relaxation consisted of a sensory-based massage with calming music in the background. In the control condition, participants listened to a music track (old-time popular music) while the experiment leader remained present. Both relaxation and alternative session had a duration of 20 minutes. After this manipulation, participants again filled in the questionnaires. Next, the exogenous cueing 
task was administered. Halfway this task, participants were offered the possibility to take a break. At the end, all participants were debriefed.

\section{Results}

\section{Group characteristics}

The groups did not differ in age, $t(35)=.01, p=.99$, gender distribution, $\chi^{2}=.23, p=$ .63 or MMSE scores, $t(35)=.49, p=.66$. Table 1 gives an overview of mean scores and standard deviations.

\section{Influence of the manipulation on VAS and PANAS}

All mean scores and standard deviations of the pre- and post-measurements of mood state can be found in Table 1. To examine whether the manipulation was successful in inducing a group difference in relaxation feelings, we performed a $2 \times 2$ mixed ANOVA with the combined VAS relaxation score as dependent variable, Time (pre- and post-scores) as within subject variables and Group (relaxation and control group) as between subjects variable. The analysis showed a main effect of time, $F(1,35)=29.28, p<.001, \eta^{2} p=.46$. More importantly, there was a significant Time $\mathrm{x}$ Group interaction effect, $F(1,35)=6.29, p=.017, \eta^{2} p=.15$. Paired samples $t$-tests were used to investigate within-group changes in VAS relaxation over time. In the relaxation group, there was a significant increase in feeling relaxed, $t(18)=6.14, p$ $<.001,95 \%$ CI $[-27.55,-13.51], d=1.87$. In contrast, in the control group, there was only a trend towards an increase in feeling relaxed, $t(17)=1.88, p=.08,95 \% \mathrm{CI}[-15.95, .90], d=.40$. Even though the groups did not differ on VAS scores before the manipulation, $t(35)=.39, p=$ .69 , independent samples $t$-tests demonstrate that the groups differ significantly on post VAS, $t(35)=2.19, p=.035,95 \%$ CI $[-21.25,-.80], d=.72$. As intended, these results indicate a larger increase in relaxation feelings in the relaxation group compared to the control group.

In a next step, we also examined whether the manipulation led to changes in mood as measured by the PANAS. A $2 \times 2$ mixed ANOVA was performed with negative mood as dependent variable, Time (pre- and post-scores) as within subject variables and Group (relaxation and control group) as between subject variable. The analysis showed a main effect of time, $F(1,35)=28.72, p<.001, \eta^{2} p=.45$. However, the Time $\mathrm{x}$ Group interaction effect was not significant, $F(1,35)=.05, p=.83$. Over the groups, there was a decrease in negative mood, $t(36)=5.44, p<.001,95 \%$ CI $[2.73,5.97], d=.82$. A similar $2 \times 2$ mixed ANOVA with positive mood as dependent variable, showed no main effect of time, $(F(1,35)=.32, p=.57$, nor an interaction effect, $F(1,35)=.59, p=.45$. However, there was a main effect of group, $F(1,35)$ $=4.52, p<.05, \eta^{2} p=.45$, pointing towards more positive mood in the relaxation group. Given 
the near significant difference between the groups for positive mood before the manipulation, $t(35)=1.92, p=.06,95 \% \mathrm{CI}[-8.67, .25], d=.63$, we conducted an univariate between-subjects ANCOVA in which we controlled for pre-scores on positive mood, with the post-scores on positive mood as dependent variable. Results showed a main effect of the pre-score of positive mood, $F(1,36)=118.05, p<.001, \eta^{2} p=.78$. However, there was no effect of group, $F(1,36)=$ $1.03, p=.32$. These results show that the manipulation succeeded in eliminating the negative mood and that positive mood remained unchanged. However, there were no group differences, indicating that the music alone manipulation was sufficient to neutralize negative feelings.

\section{Data preparation for the ECT}

Trials with errors $(M=1.75 \%)$ were omitted from analyses. In line with a previous older adult study (Demeyer \& De Raedt, 2013), responses shorter than 200ms and longer than $1250 \mathrm{~ms}$ were considered to be outliers $(M=6.34 \%)$. They respectively reflect anticipatory and delayed responding and were discarded from further analyses. These criteria resulted in the exclusion of 4 older adults ( 1 from the relaxation group, 3 from the control group) because of a loss of more than $25 \%$ of their data. Statistical analyses were performed on the remaining data.

\section{Group differences in attentional bias}

Given the significant group difference in VAS relaxation, we investigated group differences in attention. Mean reaction times and standard deviations of the reaction times for each group are presented in Table 2. The reaction times on the exogenous cueing task were subjected to a 3x 2x 2 mixed ANOVA (multivariate approach) with Cue valence (happy, neutral and sad stimuli) and Trial validity (valid and invalid) as within subject variables and Group (relaxation and control group) as between subject variable. There was a main effect of trial validity, $F(1,31)=46.56, p<.001, \eta^{2} p=.60$, indicating that all participants were faster in responding to invalid compared to valid trials. This effect is usually found in tasks using equally long cue presentation and is known as inhibition of return (Posner \& Cohen, 1984). This is the result of a suppression of the previously attended location in favor of the unattended location. This effect is indicative of the validity of the task. No other significant main or interaction effects were found (all $F<1.15, p>.33$ ).

\section{Mood-related differences in attentional bias}

We investigated mood-related differences in attentional bias. However, given the finding that there were no group differences in negative and positive mood, we investigated the relationship between mood state (as measured by post-scores on the PANAS) and attentional bias over the whole sample. First, negative mood after manipulation was used as a continuous 
factor (covariate) in a $3 \times 2$ (Cue valence $\times$ Trial validity) ANCOVA. Again, we found the significant main effect of trial validity, $F(1,31)=4.73, p<.05, \eta^{2} p=.13$. However, there were no significant interaction effects with cue valence and/or negative $\operatorname{mood}($ all $F<2.02, p>.15$ ). Secondly, positive mood after the manipulation was used as a continuous factor in a $3 \times 2$ (Cue valence $\mathrm{x}$ Trial validity) ANCOVA. We found a significant Cue valence $\mathrm{x}$ Trial validity interaction effect, $F(2,30)=3.97, p=.03, \eta^{2} p=.21$. More importantly, we found a significant 3 -way interaction effect with positive mood, $F(2,30)=4.78, p=.016, \eta^{2} p=.24$. Given that 3 separate analyses were used to investigate attentional bias, it is important to emphasize that this effect survives Bonferroni corrections.

To further investigate the interaction effect between positive mood and attention, we calculated cue validity indexes (CVI) by subtracting the mean reaction times of the valid trials from those of the invalid trials for each cue valence. Using CVI of neutral faces as a baseline, 2 new variables were constructed by subtracting CVI of neutral from both CVI for happy faces and CVI of sad faces. Pearson correlation coefficients between positive mood after manipulation and these variables were calculated. There was a significant negative correlation between positive mood and CVI (happy - neutral), $r(33)=-.49, p<.01$, indicating that participants with less positive mood showed more maintained attention to positive information compared to neutral information. For the CVI (sad - neutral), this correlation was not significant $(p=.26)$.

\section{Discussion}

In this study, we investigated the influence of a calm, relaxed mood state on attentional bias in older adults using a between-subjects experimental design. Given that inter-individual differences in mood state or stress induced by experimental paradigms may have confounded results in previous older adult studies, we used a manipulation to reduce variability in mood. One group received relaxation aimed at reducing stress and inducing feeling calm and positive and was compared to a control group listening to music.

Since previous research into the effects of relaxation has mostly used a younger sample (Sharpe et al., 2007), we first investigated the effectiveness of the manipulation. Even though both groups reported increased feelings of relaxation on the VAS scales, the manipulation was effective in inducing a larger increase in the relaxation group compared to the control group. In a next step, we investigated differences between the groups for both negative and positive mood state as measured by the PANAS. Positive mood remained unchanged throughout the experiment. For negative mood, we found a general decrease, independent of the manipulation. 
Both the relaxation procedure and the music only procedure were equally effective in alleviating negative mood. It is possible that this mood improvement in the control group resulted from the positive influence of social interaction in a population that is prone to the experience of loneliness (van Tilburg, Havens, \& Gierveld, 2004). Anyway, we could neutralize negative mood states, as intended. Moreover, the expected significant group difference on the VAS relaxation scales justified group analyses for investigating the effect on attention.

When examining group differences in attentional bias, there were no effects of the relaxation procedure on attentional bias. This might be the result of the limited effect of our manipulation on mood state. Although the groups differed in feeling relaxed, both groups showed similar positive and negative mood, which may limit the chance of finding group differences in attentional bias. Importantly, the fact that the older adults displayed a significant inhibition of return effect in general (faster to invalidly as compared to validly cued trials), testifies to the validity of the exogenous cueing task in this sample. Nevertheless, as in many other studies (e.g., Murphy \& Isaacowitz, 2008) we also failed to find indications for a general positivity bias in older adults. However, as argued before, the positivity effect in older adults may only occur under certain conditions, such as during a particular emotional state.

Therefore, in a next step, we investigated the link between post-manipulation scores on positive and negative mood and attentional bias. We found that mood was indeed related to differences in attentional processing of emotional information. Older adults who reported less positive mood showed more maintained attention towards positive information. In line with previous findings indicating a more pronounced positivity effect in older adults with elevated negative mood (e.g., Demeyer \& de Raedt, 2013, Isaacowitz et al., 2008), these results show that lower positive mood is related to a larger positivity effect. In this study, we found no relationship between attention and negative mood, which is not surprising given that our manipulation reduced the variability in negative mood. However, these results indicate that not only high negative mood, but also low positive mood may signal that more efforts are needed to achieve an optimal state of affective well-being. Consequently, individuals experiencing low positive mood would be more motivated to activate emotion regulation strategies, such as mood-incongruent preferences in attentional processing.

Given the correlational nature of this research, this finding also means that older adults who experience higher levels of positive mood show less maintained attention to positive information. One speculative explanation for this finding of increased inhibition of return for positive information (indicative of less maintained attention) is based on the notion that positive emotions can broaden cognitions and encourage exploration (Frederickson, 2001). Based on 
our findings, we might hypothesize that an encounter with positive information, which is congruent to the current mood state, might facilitate exploration to a larger extent than moodincongruent negative information, leading to more inhibition of attention to the location of the positive stimulus and exploration of the opposite location. Also Aspinwall (1998) has proposed that positive mood would signal that mood regulation is at a satisfactory level, which opens up resources to pursue goals other than mood enhancement by focussing on positive information. Thus, these findings support the idea that knowledge-related goals remain valuable in older adults at times they are not conflicting with emotion-related goals (Carstensen et al., 2003).

These results indicate that older adults also demonstrate mood-incongruent attentional preferences during positive mood, and not only during negative mood. Moreover, they confirm previous studies (e.g., Demeyer \& de Raedt, 2013, Isaacowitz et al., 2008) in demonstrating that mood state may be an important condition determining attentional processing of emotional information in older adults. More specifically, we suggest that mood may be an important condition influencing goal priorities (i.e., emotional well-being or knowledge-related goals), which in turn results in differential attentional processing of emotional information in older adults. This differential attentional processing might point towards the ability to be flexible and adapt to changing situations in older adults. Since flexibility has been associated with more psychological health and well-being (Kashdan, \& Rottenberg, 2010), the ability to adapt attentional processing might be related to older adult's resilience and increased emotional wellbeing (e.g., Carstensen et al., 2011).

Next to the limited effectiveness of our manipulation, some other limitations have to be considered. First, an important limitation is the sample. Next to its small size, the sample consisted of residential older adults. Previous research has mainly used community-dwelling older adults, who might differ in affective well-being and cognitive functioning. Given that cognitive restrains may hamper the positivity effect in attention in older adults (Reed et al., 2014), we only included participants scoring high on a screening for cognitive abilities. However, other differences between community-dwelling and rest-home residing older adults may explain our results. Second, this study did not include a younger control group. However, attentional processing of emotional information in younger adults has been well-documented (e.g., De Raedt \& Koster, 2010) and age-related differences have been established (e.g., Mather \& Carstensen, 2003).

Notwithstanding these limitations, this is, to our knowledge, the first study to show mood-incongruent attentional processing of emotional information during low positive mood in older adults. In addition to previous research showing mood-incongruent attention during 
negative mood, we found that low positive mood was related to more maintained attention to positive stimuli, which might be indicative of increased emotion regulation efforts. Based on this finding, we argued that it is important for future research to control for mood state and the resulting goal priorities since these may be important conditions determining flexible attentional processing, which may lead to more resilience in older adults. 


\section{References}

Aspinwall, L. G. (1998). Rethinking the role of positive affect in self-regulation. Motivation and Emotion, 22, 1-32. http://doi.org/10.1023/A:1023080224401

Carstensen, L. L., \& Mikels, J. A. (2005). At the intersection of emotion and cognition - Aging and the positivity effect. Current Directions in Psychological Science, 14, 117-121. http://doi.org/10.1111/j.0963-7214.2005.00348.x

Carstensen, L. L., Fung, H. H., \& Charles, S. T. (2003). Socioemotional selectivity theory and the regulation of emotion in the second half of life. Motivation and Emotion, 27, 103123. http://doi.org/10.1023/A:1024569803230

Carstensen, L. L., Isaacowitz, D. M., \& Charles, S. T. (1999). Taking time seriously - A theory of socioemotional selectivity. American Psychologist, 54, 165-181. http://doi.org/10.1037/0003-066X.54.3.165

Carstensen, L. L., Turan, B., Scheibe, S., Ram, N., Ersner-Hershfield, H., Samanez-Larkin, G. R., ... Nesselroade, J. R. (2011). Emotional experience improves with age: Evidence based on over 10 years of experience sampling. Psychology and Aging, 26, 21-33. http://doi.org/10.1037/a0021285

Cisler, J. M., \& Koster, E. H. W. (2010). Mechanisms of attentional biases towards threat in anxiety disorders: An integrative review. Clinical Psychology Review, 30, 203-216. http://doi.org/10.1016/j.cpr.2009.11.003

Deary, I. J., Corley, J., Gow, A. J., Harris, S. E., Houlihan, L. M., Marioni, R. E., .. Starr, J. M. (2009). Age-associated cognitive decline. British Medical Bulletin, 92, 135-152. http://doi.org/10.1093/bmb/ldp033

Demeyer, I., \& De Raedt, R. (2013). Attentional bias for emotional information in older adults: The role of emotion and future time perspective. Plos One, 8, e65429. http://doi.org/10.1371/journal.pone.0065429

De Clercq, A., Crombez, G., Buysse, A., \& Roeyers, H. (2003). A simple and sensitive method to measure timing accuracy. Behavior Research Methods Instruments \& Computers, 35, 109-115. http://doi.org/10.3758/BF03195502

De Raedt, R., \& Koster, E. H. W. (2010). Understanding vulnerability for depression from a cognitive neuroscience perspective: A reappraisal of attentional factors and a new conceptual framework. Cognitive Affective \& Behavioral Neuroscience, 10, 50-70. http://doi.org/10.3758/CABN.10.1.50

Folstein, M. F., Folstein, S. E., \& McHugh, P. R. (1975). 'Mini-mental state'. A practical method for grading the cognitive state of patients for the clinician. Journal of Psychiatric Research, 12, 189-198.

Fredrickson, B. L. (2001). The role of positive emotions in positive psychology - The broadenand-build theory of positive emotions. American Psychologist, 56, 218-226. http://doi.org/10.1037/0003-066X.56.3.218

Goeleven, E., De Raedt, R., Leyman, L., \& Verschuere, B. (2008). The Karolinska directed emotional faces: A validation study. Cognition \& Emotion, 22, 1094-1118. http://doi.org/10.1080/02699930701626582

Isaacowitz, D. M., \& Blanchard-Fields, F. (2012). Linking process and outcome in the study of emotion and aging. Perspectives on Psychological Science, 7, 3-17. http://doi.org/10.1177/1745691611424750

Isaacowitz, D. M., Allard, E. S., Murphy, N. A., \& Schlangel, M. (2009). The time course of age-related preferences toward positive and negative stimuli. Journals of Gerontology Series B- Psychological Sciences and Social Sciences, 64, 188-192. http://doi.org/10.1093/geronb/gbn036 
Isaacowitz, D. M., Toner, K., Goren, D., \& Wilson, H. R. (2008). Looking while unhappy mood-congruent gaze in young adults, positive gaze in older adults. Psychological Science, 19, 848-853. http://doi.org/10.1111/j.1467-9280.2008.02167.x

Isaacowitz, D. M., Wadlinger, H. A., Goren, D., \& Wilson, H. R. (2006). Is there an age-related positivity effect in visual attention? A comparison of two methodologies. Emotion, 6, 511-516. http://doi.org/10.1037/1528-3542.6.3.511

Kashdan, T. B., \& Rottenberg, J. (2010). Psychological flexibility as a fundamental aspect of health. Clinical Psychology Review, 30, 865-878. http://doi.org/10.1016/j.cpr.2010.03.001

Koster, E. H. W., De Raedt, R., Goeleven, E., Franck, E., \& Crombez, G. (2005). Moodcongruent attentional bias in dysphoria: Maintained attention to and impaired disengagement from negative information. Emotion, 5, 446-455. http://doi.org/10.1037/1528-3542.5.4.446

Mather, M., \& Carstensen, L. L. (2003). Aging and attentional biases for emotional faces. Psychological Science, 14, 409-415. http://doi.org/10.1111/1467-9280.01455

Mather, M., \& Carstensen, L. L. (2005). Aging and motivated cognition: The positivity effect in attention and memory. Trends in Cognitive Sciences, 9, 496-502. http://doi.org/10.1016/j.tics.2005.08.005

Murphy, N. A., \& Isaacowitz, D. M. (2008). Preferences for emotional information in older and younger adults: A meta-analysis of memory and attention tasks. Psychology and Aging, 23, 263-286. http://doi.org/10.1037/0882-7974.23.2.263

Peeters, F. P. M. L., Ponds, R. W. H. M., \& Vermeeren, M. T. G. (1996). Affectiviteit en zelfbeoordeling van depressie en angst [affectivity and self evaluation of depression and anxiety]. Tijdschrift voor Psychiatrie, 38, 240-250.

Posner, M. I. (1980). Orienting of attention. Quarterly Journal of Experimental Psychology, 32, 3-25. http://doi.org/10.1080/00335558008248231

Posner, M. I., \& Cohen, Y. (1984). Components of visual orienting. In H. Bouma \& D. Bouwhuis (Eds.), Attention perform, (Vol. X., pp. 531-556). Hove, UK: Erlbaum.

Reed, A. E., Chan, L., \& Mikels, J. A. (2014). Meta-analysis of the age-related positivity effect: Age differences in preferences for positive over negative information. Psychology and Aging, 29, 1-15. http://doi.org/10.1037/a0035194

Sharpe, P. A., Williams, H. G., Granner, M. L., \& Hussey, J. R. (2007). A randomised study of the effects of massage therapy compared to guided relaxation on well-being and stress perception among older adults. Complementary Therapies in Medicine, 15, 157-163. http://doi.org/10.1016/j.ctim.2007.01.004

Steinmetz, K. R. M., Muscatell, K. A., \& Kensinger, E. A. (2010). The effect of valence on young and older adults' attention in a rapid serial visual presentation task. Psychology and Aging, 25, 239-245. http://doi.org/10.1037/a0018297

Tombaugh, T. N., \& McIntyre, N. J. (1992). The mini-mental state examination - A comprehensive review. Journal of the American Geriatrics Society, 40, 922-935. http://doi.org/10.1111/j.1532-5415.1992.tb01992.x

Urry, H. L., \& Gross, J. J. (2010). Emotion regulation in older age. Current Directions in Psychological Science, 19, 352-357. http://doi.org/10.1177/0963721410388395

van Tilburg, T., Havens, B., \& Gierveld, J. D. (2004). Loneliness among older adults in the Netherlands, Italy, and Canada: A multifaceted comparison. Canadian Journal on Aging, 23, 169-180. http://doi.org/10.1353/cja.2004.0026

Watson, D., Clark, L. A., \& Tellegen, A. (1988). Development and validation of brief measures of positive and negative affect - The PANAS scales. Journal of Personality and Social Psychology, 54, 1063-1070. http://doi.org/10.1037/0022-3514.54.6.1063 
Table 1.

Mean scores and standard deviations for all measurements in the relaxation and control group.

\begin{tabular}{lrrrrrrrrr}
\hline & \multicolumn{3}{c}{ relaxation group } & \multicolumn{4}{c}{ control group } \\
& \multicolumn{2}{c}{ pre } & \multicolumn{2}{c}{ post } & \multicolumn{2}{c}{ pre } & \multicolumn{2}{c}{ post } \\
& $M$ & $S D$ & $M$ & $S D$ & $M$ & $S D$ & $M$ & $S D$ \\
\hline Age & 80.53 & 8.70 & & & 80.50 & 6.56 & & \\
MMSE & 25.74 & 1.56 & & & 26.00 & 1.99 & & \\
PA & 34.21 & 6.46 & 34.95 & 6.24 & 30.00 & 6.89 & 29.89 & 7.70 \\
NA & 17.89 & 4.73 & 13.37 & 4.28 & 17.61 & 6.29 & 13.44 & 5.73 \\
VAS & 64.15 & 10.97 & 84.68 & 11.00 & 66.12 & 18.52 & 73.65 & 18.83 \\
\hline
\end{tabular}

Note . MMSE = Mini Mental State Examination; PA = positive affect; NA = negative affect; VAS = Visual Analogue Scale. 
Table 2. Mean reaction times (in ms) and standard deviations as a function of Cue valence and Trial Validity in the relaxation and control group.

\begin{tabular}{cccccc}
\hline & & \multicolumn{2}{c}{ Relaxation group } & \multicolumn{2}{c}{ Control group } \\
Cue Valence & Trial Validity & $M$ & $S D$ & $M$ & $S D$ \\
\hline \multirow{2}{*}{ happy } & valid & 681 & 144 & 694 & 147 \\
& invalid & 613 & 143 & 642 & 144 \\
\multirow{2}{*}{ neutral } & valid & 659 & 148 & 697 & 138 \\
& invalid & 614 & 146 & 638 & 147 \\
\multirow{2}{*}{ sad } & valid & 670 & 147 & 694 & 152 \\
& invalid & 616 & 148 & 638 & 143 \\
\hline
\end{tabular}

